\title{
Approaches for arthroscopic management in partial articular rotator cuff tears - literature review
}

\author{
Volume 8 Issue I - 2017 \\ León Ezagui Bentolila \\ Hospital Universitari de Bellvitge, Spain
}

\section{Introduction}

Partial articular sided rotator cuff tears represent a common source of shoulder pain and disability, with articular sided tears being 2 to 3 times more common than bursal sided tears, ${ }^{1}$ and affecting predominantly the supraspinatus tendon, ${ }^{2}$ which has an insertion footprint that averages $16 \mathrm{~mm}$ in width in the medial to lateral dimension. ${ }^{3}$ On the basis of this anatomic feature, Elman was the first author to describe a classification system that separated articular sided tears into 3 grades depending on the amount of medial to lateral dimension of tendon thickness that is interrupted, as measured during arthroscopic evaluation. Grade 1: less than $3 \mathrm{~mm}$, grade 2: 3-6 mm and grade 3 exceeds $6 \mathrm{~mm}$, intended to represent roughly more than $50 \%$ of tendon thickness. ${ }^{1}$

Habermeyer et al. ${ }^{4}$ in their efforts to find a descriptive rationale to quantify articular sided supraspinatus tendon tears in the transverse and coronal planes, leading to a 2 dimensional description of the tear pattern, described their classification assessed by the length of the peeled-off bony footprint in the coronal plane and by the sagittal tear extension in the transverse plane. Regarding to the coronal plane, a type 1 tear is a small tear within the transition zone from cartilage to bone, a type 2 tear is defined as a tear up to the center of the footprint, and a type 3 tear extends up to the greater tuberosity. The sagittal tear extension in the transverse plane is defined as a tear of the lateral reflection pulley on the medial border of the supraspinatus tendon and/ or a tear in the area of the crescent zone. A tear of the lateral portion of the coracohumeral ligament continuing into the medial border of the supraspinatus tendon is defined as a type A tear, an isolated partialthickness tear of the supraspinatus tendon within the crescent zone is a type B tear, and a tear extending from the lateral portion of the coracohumeral ligament and continuing into the medial border of the supraspinatus tendon up to the crescent zone is defined as a type $\mathrm{C}$ tear.

After failed conservative management; operative intervention is typically indicated for patients with persistent pain and disability symptoms. The etiology of these tears is often multifactorial and can be related to outlet impingement, shoulder instability, trauma, repetitive overhead throwing, or intrinsic tendon degeneration or a combination of these factors, and successful management of patients with Partial thickness rotator cuff tears requires treatment of the tendon itself and any associated pathology. A high incidence of associated pathology in this patient group has been found, and evidenced by the large number of associated procedures usually performed concomitantly with the rotator cuff repair. ${ }^{5}$

A variety of approaches for arthroscopic management of partial-thickness rotator cuff tears have been reported, including acromioplasty alone, debridement of the partial-thickness tear with or without acromioplasty, trans-tendinous repair, or conversion of the lesion to a full-thickness tear followed by repair.

Multiple studies have described poor results with debridement of Partial lesions of the rotator cuff comprising greater than $50 \%$
Correspondence: Leon Ezagui Bentolila, Hospital Universitari de Bellvitge, Barcelona, Spain, Email lezagui8@gmail.com

Received: April II, 2017| Published: April 21, 2017

of the thickness of the footprint, ${ }^{6}$ and although the current practice is to debride partial-thickness tears comprising $<50 \%$ of the tendon thickness and to repair high-grade tears $>50 \%$, data supporting one particular management approach for this type of tears are variable and limited, and on the basis of the available evidence no single repair technique provides superior clinical outcomes. ${ }^{7}$ Still significant controversy exists regarding the most appropriate method to surgically repair Partial articular-sided cuff tears.

The arthroscopic Trans tendon repair was first proposed by Snyder and later by Lo \& Burkhart. ${ }^{8}$ The purpose of this technique is to avoid removing residual bursal-sided cuff tissue to complete the tear and to repair only the deeper portion of the cuff to the greater tuberosity. In this manner, the medial aspect of the footprint is restored, while preserving the intact lateral tendon. This re-establishment of the normal rotator cuff footprint potentially minimizes any length-tension mismatch of the repaired cuff.

In this technique, as described by Lo and Burkhart, an arthroscope is introduced into the glenohumeral joint through a posterior portal and the partial-thickness rotator cuff tear is observed. To place transtendon anchors, they initially used an 18-gauge spinal needle as a guide. The angle of insertion is usually directly adjacent to the lateral aspect of the acromion, allowing the anchor to be placed at a dead man's angle of $45^{\circ}$ or less into the medial margin of the rotator cuff footprint. A punch is introduced through a percutaneous 3-mm stab incision partial to the 18-gauge spinal needle, penetrating the rotator cuff and creating a bone socket for the anchor. By maintaining the same angle of insertion, a suture anchor is then placed transtendon into the bone socket. If the bare footprint is large enough in its anterior-to-posterior dimension, they placed a second transtendon anchor approximately $1 \mathrm{~cm}$ posterior to the first anchor. For a 1-anchor repair, the author explained the necessity to create a bridge of tissue to compress against the bone bed. To accomplish this, they used a Penetrator (Arthrex) or Bird Beak (Arthrex) suture passer to pass one limb of each suture retrograde through a more posterior area of the tear, and then tying the sutures in a routine fashion in the subacromial space. Multiples descriptions and variations of the technique has been published, and all reported a reliable 
In 2005, Ide et al. ${ }^{9}$ reported clinical results for the trans-tendon technique in 17 patients observed for a mean follow-up of 39 months. The mean UCLA and JOA scores improved significantly from 17.3 to 68.4 points to 32.9 and 94.8 points, respectively. Also in 2005, Waibl $\&$ Buess ${ }^{10}$ reported clinical results of this technique in 22 patients who were observed for a mean follow-up of 16 months. The mean UCLA score improved significantly from 17.1 points to 31.2 .

In 2008, Tauber et al. ${ }^{11}$ described a transtendon arthroscopic technique of transosseous refixation in patients with Ellman grade 2 and 3 articular-side Partial tears, leaving the bursal layer of

the supraspinatus tendon intact. A curved hollow needle is used to perform an all arthroscopic transosseous mattress suture, thus anatomical tendon-to-bone contact of the rotator cuff to the footprint is restored. Preliminary clinical results of 16 patients were convincing with significant pain relief and functional improvement, with a mean UCLA score improved significantly from 15.8 points preoperatively to 32.8 points at follow-up $(\mathrm{P}<0.01)$. Evaluation of pain using the VAS also showed significant improvement from 7.9 points before surgery to 1.2 points post-operatively $(\mathrm{P}<0.01)$.

In 2009, Castagna et al. ${ }^{12}$ reported clinical results in 54 patients observed for a mean follow-up of 32.4 months. The mean UCLA and Constant-Murley scores improved significantly from 14.1 to 45.3 points to 32.9 and 90.6 points, respectively. Also in 2009, Castricini et al. ${ }^{13}$ reported clinical results in 31 patients observed for a mean follow-up of 33 months. The mean Constant-Murley score improved significantly from 44.4 points to 91.6 points.

In 2010 Spencer, ${ }^{14}$ in his concern regarding the transtendinous techniques that can be associated with stiffness and also knowing that completing the tear takes down normal intact tissue, developed a technique that repairs the articular-side partial- thickness rotator cuff tears with an all-inside approach that does not violate the intact bursal tissue and does not complete the tear.

In this technique, an anchor is placed through the intact bursal portion of the rotator cuff into the medial portion of the greater tuberosity, which is first localized with a spinal needle. The author usually uses a 3-mm polyetheretherketone anchor to minimize the size of the entry site. The sutures are then brought into the joint. A suture passing device is placed in the lateral portal and then passed through just the articular layer of the rotator cuff. The wire from the suture passing device and a suture limb are retrieved through an inferior cannula. The suture is placed in the wire and pulled through the tissue. This passes the suture through the articular lamina of the rotator cuff. The other limb of the suture is then retrieved into the lateral cannula so that both limbs are within the lateral cannula and ready to be tied. The articular layer is then tied down, with the knot getting buried between the intact bursal layer and the newly repaired articular layer.

The clinical results reported in 20 patients observed for a mean follow-up of 29 months, using the mean Penn Shoulder Score improved significantly from 74 points to 92 points.

In 2012, Duralde $\mathrm{X}$ et al. ${ }^{5}$ reported clinical results after retrospectively evaluating 53 patients who underwent arthroscopic transtendinous repair for Ellman grade III articular-sided rotator cuff tears, showing that the American Shoulder and Elbow Surgeons scores improved from a mean of 48.0 to $89.4(+41.4)(\mathrm{P}<.0001)$. Pain scores on a visual analog scale improved from 5.7 to $1.0(\mathrm{P}<.0001)$. Ninetyeight percent of patients were satisfied with the results of surgery. And results for the 50 patients available for follow-up were excellent in 32 $(64 \%)$, good in $6(12 \%)$, fair in $6(12 \%)$, and poor in $6(12 \%)$.
Kim K et al. ${ }^{15}$ published the functional and radiological outcomes of arthroscopic trans-tendon suture-bridge repair. In this technique, one or two bio-absorbable suture anchors are inserted at the articular margin to create a medial row through the intact cuff. Then, no. 2 polydioxanone (PDS) sutures are advanced through each spinal needle, and a grasper is used to shuffle these sutures out of an anterior cannula. The anchor sutures are tied to the PDS and pulled into the joint, and the knots are shuttled externally by pulling on the percutaneously located PDS sutures. Then, the arthroscope is moved to the subacromial space. The strands of the anchor are tied with a sliding knot, but the sutures are not cut. Pilot holes for a second anchor are prepared directly in line with the medial anchors and approximately $5-10 \mathrm{~mm}$ distal to the lateral edge of the greater tuberosity. The second anchor is advanced into the pilot hole completely, and these steps are repeated for a second construct.

For this technique, the autors reported a mean UCLA, ASES and Constant-Murley scores improve from $19.1 \pm 5.4,45.2 \pm 16.0$ and $58.0 \pm 19.6$ preoperatively to $35.7 \pm 8.5,79.0 \pm 15.8$ and 78.1 \pm 12.9 at final follow-up, respectively (all $\mathrm{p}<0.001$ ). Mean VAS score and ROM (forward flexion) improved from $6.1 \pm 1.9$ and 140 \pm 36.6 preoperavely to $2.6 \pm 1.9$ and $163 \pm 25.2$ at the final followup, respectively $(p<0.001)$. Additionally they described that the postoperative radiological examination showed cuff integrity without retear in all patients.

When comparing the transtendinous repair technique and conversion to a full thickness tear, both repairing techniques provide good results in terms of function and pain, and no statically significant deference's between the two techniques has been found in postoperative comparative studies. ${ }^{16,17}$

An interesting difference described by $\operatorname{Shin}^{18}$ is that although repair after conversion to a full-thickness tear shows less postoperative morbidity, tendon integrity is of primary concern after repair, and on the other hand, the transtendon repair technique results in complete tendon integrity but slower functional recovery. Also in a cadaveric study, Sethi et al. ${ }^{19}$ described that in situ repair is a viable biomechanical treatment option compared with tear completion prior to repair of Partial articular-sided rotator cuff tears, and when clinically appropriate, the in situ repair may offer some biomechanical advantages, with lower strain and displacement observed on the bursal side compared with tear completion prior to repair. Biomechanical studies have also shown that the transtendinous technique of repair is biomechanically superior to a double-row technique in terms of both ultimate failure strength and gap formation. ${ }^{20}$

More recently, Castagna et al. $^{21}$ compared the clinical and subjective difference between transtendon repair or arthroscopic completion of the tear and formal repair. The study showed a Constant score improve by a mean value of $25(95 \%$ CI $21-28)(p<0.0001)$ and of $29(95 \%$ CI 26-31) $(\mathrm{p}<0.0001)$, respectively, and a VAS score decrease of $3.4(95 \%$ CI 2.9-3.9) $(\mathrm{p}<0.0001)$ and of $3.6(95 \% \mathrm{CI}$ $3.3-4.0)(p<0.0001)$, respectively. With these results they report that both repairing techniques of deep Partial supraspinatus tear provide good results in terms of function and pain. There were no statistically significant deference's between the two techniques.

\section{Conclusion}

As shown, the literature describes that arthroscopic transtendinous repair of Partial articular-sided rotator cuff tears is a safe and effective treatment that allows identification of commonly associated pathology and reliable improvement in pain and function, and when compared to conversion to a full thickness tear, most reports agree that both 
procedures are safe, effective, and comparable for clinical, functional and MRI findings.

\section{Acknowledgments}

None.

\section{Conflicts of interest}

None.

\section{References}

1. Ellman H. Diagnosis and treatment of incomplete rotator cuff tears. Clin Orthop Relat Res. 1990;254:64-74.

2. Fukuda $H$. The management of Partialthickness tears of the rotator cuff. J Bone Joint Surg. 2003;85B:3-11

3. Curtis AS, Burbank KM, Tierney JJ, et al. The insertional footprint of the rotator cuff: an anatomic study. Arthroscopy. 2006;22(6):609 e1.

4. Habermeyer P, Krieter C, Tang KL, et al. A new arthroscopic classificaon of articular sided supraspinatus footprint lesions: A prospecve comparison with Snyder's and Ellman's classification. J Shoulder Elbow Surg. 2008;17(6):909-913.

5. Duralde X, McClelland W. Transtendinous repair of supraspinatus tears. Arthroscopy. 2012;28(2):160-168.

6. Weber SC. Arthroscopic debridement and acromioplasty versus miniopen repair in the treatment of significant Partialthickness rotator cuff tears. Arthroscopy. 1999;15(2):126-131

7. Bollier M, Shea K. Systemac Review: What surgical technique provides the best outcome for symptomac Partialarticular sided rotator cuff tears? The Iowa Orthopaedic Journal. 2012;32:164-172.

8. Lo IK, Burkhart SS. Transtendon arthroscopic repair of Partialthickness, articular surface tears of the rotator cuff. Arthroscopy. 2004;20(2):214-220.

9. Ide J, Maeda S, Takagi K. Arthroscopic transtendon repair of partialthickness arcular-side tears of the rotator cuff: anatomical and clinical study. Am J Sports Med. 2005;33(11):1672-1679.
10. Waibl B, Buess E. Partial-thickness articular surface supraspinatus tears: a new transtendon suture technique. Arthroscopy. 2005;21:376-381.

11. Tauber M, Koller H, Resch H. Transosseous arthroscopic repair of Partialarcular-surface supraspinatus tendon tears. Knee Surg Sports Traumatol Arthrosc. 2008;16(6):608-613.

12. Castagna A, Delle Rose G, Con M, et al. Predicve factors of subtle residual shoulder symptoms aer transtendinous arthroscopic cuff repair: a clinical study. Am J Sports Med. 2009;37(1):103-108

13. Castricini R, Panfoli N, Nioli R, et al. Transtendon arthroscopic repair of partial-thickness, articular surface tears of the supraspinatus: results at 2 years. Chir Organi Mov. 2009;93(Suppl 1):S49- S54

14. Spencer EE. Partial-thickness articular surface rotator cuff tears: an allinside repair technique. Clin Orthop Relat Res. 2010;468(6):1514-1520.

15. Kim K, Shin H, Min Cha S, et al. Clinical outcomes after arthroscopic trans-tendon suture-bridge technique in Partialthickness arcular-side rotator cuff. Knee Surg Sports Traumatol Arthrosc. 2013;21:1183-1188.

16. Castagna A1, Borroni M, Garofalo R, et al. Deep Partialrotator cuff tear: transtendon repair or tear completion and repair? A randomized clinical trial. Knee Surg Sports Traumatol Arthrosc. 2015;23(2):460-463.

17. Franceschi F, Papalia R, Buono AD, et al. Arcular-sided rotator cuff tears: which is the best repair? A three-year prospecve randomised controlled trial. Int Orthop. 2013;37:1487-1493.

18. Shin S () A Comparison of 2 Repair Techniques for Partial-Thickness Arcular-Sided Rotator Cuff Tears. Arthroscopy: The Journal of Arthroscopic and Related Surgery Vol 28(1): 25-33.

19. Sethi PM, Rajaram A, Obopilwe E, et al. PartialArcular-sided Rotator Cuff Tears: In Situ Repair Versus Tear Compleon Prior to Repair. Orthopedics. 2013;36(6):771-777.

20. Gonzalez-Lomas G, Kippe MA, Brown GD, et al. In situ transtendon repair outperforms tear compleon and repair for Partialarcular-sided supraspinatus tendon tears. J Shoulder Elbow Surg. 2008;17(5):722-728.

21. Castagna A, Borroni M, Garofalo R, et al. Deep partial rotator cuff tear: transtendon repair or tear completion and repair? A randomized clinical trial. Knee Surg Sports Traumatol Arthrosc. 2015;23(2):460-463. 